
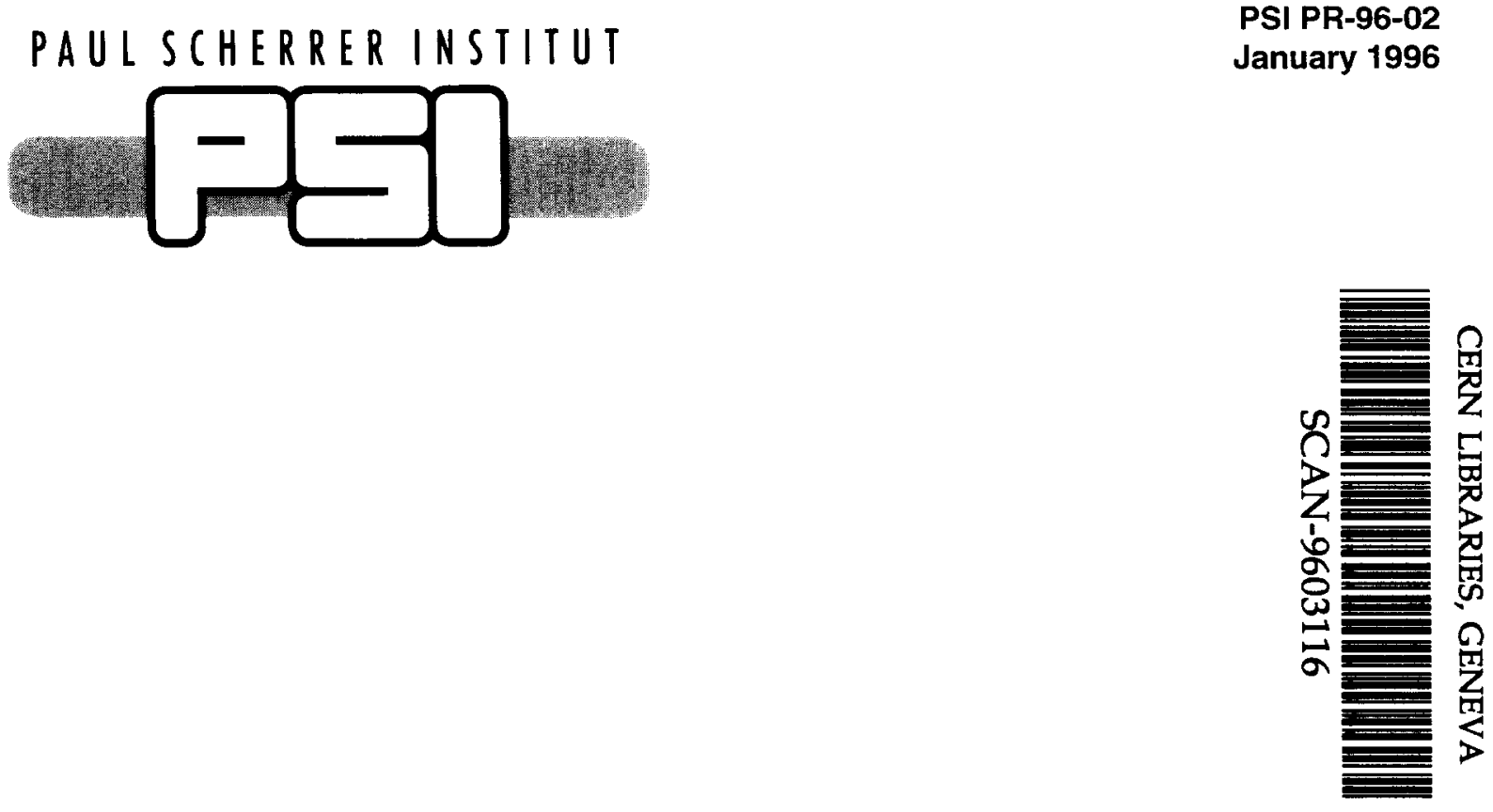

$5 \operatorname{cog} 611$

\title{
Baryonic Dark Clusters in Galactic Halos
}

F. De Paolis ${ }^{1}$, G. Ingrosso ${ }^{1}$, Ph. Jetzer², M. Roncadelli ${ }^{3}$

1 Dipartimento di Fisica, Università di Lecce, CP 193, 1-73100 Lecce, Italy and INFN, Sezione di Lecce, CP 193, 1-73100 Lecce, Italy

2 Paul Scherrer Institut, Laboratory for Astrophysics, $\mathrm{CH}-5232$ Villigen PSI, Switzerland and Institute of Theoretical Physics, University of Zürich, Winterthurerstrasse 190 $\mathrm{CH}-8057$ Zürich, Switzerland

3 INFN, Sezione di Pavia, Via Bassi 6, 1-27100 Pavia, Italy 


\title{
Baryonic Dark Clusters in Galactic Halos
}

\author{
F. De Paolis ${ }^{1}$, G. Ingrosso ${ }^{1}$, Ph. Jetzer ${ }^{2}$ and M. Roncadelli ${ }^{3}$ \\ ${ }^{1}$ Dipartimento di Fisica, Università di Lecce, CP 193, 73100 Lecce, Italy \\ and INFN, Sezione di Lecce, CP 193, 73100 Lecce, Italy \\ ${ }^{2}$ Paul Scherrer Institut, Laboratory for Astrophysics, \\ CH-5232 Villigen PSI, Switzerland \\ and Institute of Theoretical Physics, University of Zurich, \\ Winterthurerstrasse 190, CH-8057 Zurich, Switzerland \\ ${ }^{3}$ INFN, Sezione di Pavia, Via Bassi 6, I-27100, Pavia
}

\begin{abstract}
Besides MACHOs (Massive Astrophysical Compact Halo Objects) discovered by microlensing, cold molecular clouds (mainly of $\mathrm{H}_{2}$ ) may well contribute substantially to the galactic halo dark matter. We describe a model for the formation and evolution of proto globular clusters towards either globular clusters or dark clusters of MACHOs and molecular clouds, depending on the distance from the galactic centre. Moreover, we discuss various methods to test this scenario, which relay upon observations in several bands of the electromagnetic spectrum. In particular, we estimate the $\gamma$-ray flux arising from halo molecular clouds through the interaction with high-energy cosmic-ray protons. Molecular clouds can also be detected via the absorption lines they would produce in the spectrum of stars located in the Large Magellanic Cloud and via the anisotropy they would introduce in the Cosmic Background Radiation when looking at the halo of M31 galaxy. Finally, we address the possibility of discovering MACHOs by infrared searches.
\end{abstract}

Invited paper to appear in International Journal of Modern Physics D. . 


\section{Introduction}

One of the most important problems in astrophysics concerns the nature of the dark matter in galactic halos, whose presence is implied by the observed flat rotation curves in spiral galaxies $[1,2,3,4,5]$. As is well known, primordial nucleosynthesis [6] entails that most of the baryonic matter in the Universe is nonluminous. And such an amount of dark matter falls suspiciously close to that required by the rotation curves. Surely, the standard model of elementary particle forces can hardly be viewed as the ultimate theory and all the attempts in that direction invariably call for new particles. Hence, the idea of nonbaryonic dark matter naturally enters the realm of cosmology and may help to understand the process of galaxy formation and clustering of galaxies. As far as the baryonic component of the Galaxy is concerned, collapsed objects (black holes and neutron stars) are excluded as candidate halo constituents by the observed low metallicity of the halo, except for super-massive black holes. With this exception, the baryonic constituents of the halo should be in the form of MACHOs with masses in the range $10^{-7}<M / M_{\odot}<10^{-1}[7]$, which can be detected - as proposed by Paczyński - via the gravitational lens effect $[8,9,10,11]$. A further possibility is provided by gas clouds sufficiently cold to have escaped detection.

\subsection{Motivation and goals}

Since September 1993 the French collaboration EROS [12, 13] and the American-Australian collaboration MACHO [14] announced the detection of a few microlensing events, discovered by monitoring over several years millions of stars in the Large Magellanic Cloud (LMC) ${ }^{1}$. In addition, the Polish-American collaboration OGLE [15], the MACHO [16] and the French DUO [17] collaborations found altogether more than $\sim 100$ microlensing events by monitoring stars located in the galactic bulge. The inferred optical depth for the bulge is considerably higher than previously thought $[18,19]$. This circumstance can be explained if the mass of the disk is larger than assumed in the standard galactic models and/or if the galactic bulge has the form of a bar pointing nearly along the line of sight $[20,21]$.

The microlensing events found so far towards the LMC do not yet permit to make a precise estimate of the fraction of halo dark matter in the form of MACHOs, nor to infer whether

\footnotetext{
${ }^{1}$ Five events altogether have been reported in published papers, but we understand that more events have been recorded by the MACHO collaboration in the analysis of their second year data.
} 
they are located inside the halo or rather either in the LMC itself or in the spheroid or in a thick disk of our galaxy $[22,23,24,25,26,27]$. Nevertheless, assuming a standard spherical halo model, Alcock et al. [28] have found (analyzing their first year data) that MACHOs contribute with a fraction $\eta=0.19_{-0.10}^{+0.16}$ to the halo dark matter ${ }^{2}$, whereas their average mass turns out to be $\sim 0.08 M_{\odot}$ [30]. The EROS collaboration was able to set stringent limits on the presence of MACHOs in the mass range $5 \times 10^{-8}<M / M_{\odot}<5 \times 10^{-4}$ inside the halo [31].

Thus, the problem arises to explain the formation of MACHOs, as well as the nature of the remaining amount of dark matter in galactic halos.

We feel it hard to conceive a formation mechanism which transforms with $100 \%$ efficiency hydrogen and helium gas into MACHOs. Therefore, we expect that also cold clouds should be present in the galactic halo.

We have proposed a scenario $[32,33,34]$ in which dark clusters ${ }^{3}$ of MACHOs and cold molecular clouds - mainly of $\mathrm{H}_{2}$ - naturally form in the halo at galactocentric distances larger than $10-20 \mathrm{kpc}$. A similar scenario has also been put forward by Gerhard and Silk [40].

Pfenniger, Combes and Martinet [41] have instead suggested that $H_{2}$ molecular clouds can constitute the dark matter in the disk of our galaxy. They point out that a much larger amount of cold gas in the outer disk of spiral galaxies could help to understand their evolution along the Hubble sequence from Sd to Sa over some billions of years. In fact, the varying $M / L$ ratio along the Hubble sequence suggests that the dark matter is transformed into stars, thereby implying that the dark matter should be baryonic and in the form of gas. In this scenario the $\mathrm{H}_{2}$ molecular clouds have a fractal structure that ranges upwards over 4 to 6 orders of magnitude in scale. The elementary cloudlets have low temperature $\sim 3 \mathrm{~K}$, typical number density $\sim 10^{9} \mathrm{~cm}^{-3}$, size $\sim 5 \times 10^{-6} \mathrm{pc}$ and mass $\sim 10^{-3} M_{\odot}$. They are self-gravitating and their individual thermal width for $H_{2}$ along the line of sight is about $0.1 \mathrm{~km} \mathrm{~s}^{-1}$.

Recently, Bahcall, Lubin and Dorman [42] have claimed that most of the dark matter in

\footnotetext{
${ }^{2} \mathrm{~A}$ recent evaluation of the microlensing rates by using more general, self-consistent models for the distribution of luminous and dark matter (with radial anisotropy in velocity space) in the Galaxy shows that the above-quoted fraction $\eta$ of the dark matter in the form of MACHOs can increase by a factor up to 1.5 [29].

${ }^{3}$ The possibility that MACHOs are clumped into dark clusters has also been investigated by several authors (see e.g. [35, 36, 37, 38, 39]).
} 
galaxies and clusters of galaxies should reside in very large halos around galaxies, typically extending up to $\sim 200 \mathrm{kpc}$ for bright galaxies. The total mass of large-scale systems such as rich clusters of galaxies and even superclusters can be fully accounted for by the total mass of their member galaxies, including their halos.

The presence of cold gas at very low temperature at the centre of both elliptical galaxies and clusters of galaxies has also been inferred via X-ray observations of cooling flows (for a recent review see [43]). For instance, in bright ellipticals, where hot diffuse gas at a temperature of $\sim 10^{7} \mathrm{~K}$ is observed, cooling reduces the gas temperature at the centre of the galaxy and the only way for the gas to restore equilibrium (inside the gravitational potential well) is to flow inward. Hence, the inner gas drops out forming cold clouds throughout the flow, thereby resulting in some form of dark matter. In fact, in the lack of any observations of cooling flows apart from the X-ray band, one is led to imagine that a certain fraction of the cold gas forms invisible low-mass stars (with an upper mass limit of $\sim 0.2 M_{\odot}$ ), while the remaining gas gives rise to dark molecular clouds at a temperature near to that of the Cosmic Background Radiation (CBR) (see [43] and section 3.2). A similar picture should also holds for the central regions of clusters of galaxies, where the cold gas falls into the halo of the central galaxy.

The detection of cold molecular clouds is very difficult. Indeed, their presence in the disk of the Galaxy, where the clouds have been enriched by metals, is inferred mainly through $\mathrm{CO}$ emission. From the $\mathrm{CO}$ abundance one can estimate the amount of $\mathrm{H}_{2}$ by assuming a constant $\mathrm{CO} / \mathrm{H}_{2}$ ratio. However, this ratio depends on the metallicity. So, it might well be that in clouds located either in the outer part of the disk or inside the halo of our galaxy this ratio is smaller due to a lower metallicity. Therefore, it would be even more difficult to detect such clouds there. Nevertheless, Lequeux, Allen and Guilloteau [44] reported the detection of faint $C O$ lines from gas at a distance of about $12 \mathrm{kpc}$ from the galactic centre. This observation suggests that there is a significant amount of $H_{2}$ gas in the outer regions of the galactic disk.

Furthermore, radioastronomical observations have revealed the existence of radio signals from galaxy-sized clouds of $C O$ in several damped Lyman- $\alpha$ absorbers at redshifts between 2 and 3.2 [45]. A fact this, if confirmed, which entails that the formation of dense molecular clouds can already occur at a very early stage in the evolution of galaxies. Quite recently, 
Weiner and Williams [46] have detected a faint Lyman- $\alpha$ emission from several points along the Magellanic Stream, which hints at the presence of a relatively large density of $H I$ in the galactic halo at $\sim 50 \mathrm{kpc}$ and far above the galactic plane. This would imply that the galaxy has a very large gaseous extent.

\subsection{Outlook}

We argue that the halo of our galaxy contains dark clusters of MACHOs and cold molecular clouds (mainly of $\mathrm{H}_{2}$ ), with relative abundance depending on the galactocentric distance. In section 2 , we discuss this scenario within the framework of the protocluster cloud model for the galactic evolution, addressing several specific items. Section 3 is devoted to various observational tests of our picture. We estimate the $\gamma$-ray flux arising from halo molecular clouds through the interaction with high-energy cosmic-ray protons. Molecular clouds can also be detected via the absorption lines they would produce in the spectrum of stars located in the LMC and via the anisotropy they would introduce in the CBR when looking at the halo of M31 galaxy. Finally, we address the possibility of discovering MACHOs by infrared searches. In section 4 we offer our conclusions.

We would like to emphasize that the present paper should be understood more as a status report than as a review article: while some topics are treated in detail others are merely touched upon, mainly because they are still rather unsettled.

\section{Scenario}

\subsection{Cluster formation mechanism}

Our scenario for the formation of dark clusters of MACHOs and cold molecular clouds encompasses the one first proposed by Fall and Rees [47] (and considered in a more detailed form by Kang, Shapiro, Fall and Rees [48]) and makes use of the bimodal star formation process (via thermal or gravitational instability) advocated by Murray and Linn [49]. Our picture also relies upon the suggestion of Palla, Salpeter and Stahler [50] that the lower bound on the fragment masses in a collapsing, metal poor cloud can be as low as $10^{-2} M_{\odot}$.

Let us begin by summarizing the ideas of Fall and Rees [47] from a point of view which is most convenient for our considerations. 
After the initial collapse, the proto galaxy $(\mathrm{PG})$ is expected to reach a quasi-hydrostatic equilibrium state with virial temperature $\sim 10^{6} \mathrm{~K}$. Fall and Rees [47] have shown that in such a situation a thermal instability develops: density enhancements rapidly grow as the gas cools to lower temperatures. In fact, irregularities in the inflow during the gas collapse and also fluctuations in the distribution of nonbaryonic dark matter (if present on the galactic scale) would introduce perturbations with a wide range in size and amplitude. As a result, randomly distributed overdense regions will form inside the $\mathrm{PG}$. For reasons that will become clear later, these overdense regions will be referred to as proto globular cluster (PGC) clouds.

Under the assumption that the plasma in the PG is in ionization equilibrium - namely that it recombines faster than it cools - it turns out that the cooling rate (as a function of density $\rho$ and temperature $T$ ) has the form [47]

$$
\Lambda(\rho, T)=\rho^{2} L(T)
$$

(the expression of $L(T)$ can be found e.g. in [51]). The cooling time is

$$
t_{\text {cool }}=\frac{3 \rho k_{B} T}{2 \mu(\Lambda-\Gamma)}
$$

where the heating rate $\Gamma$ due to external heating sources has been taken into account (here $\mu \sim 1.22 m_{p}$ is the mean molecular mass of the primordial gas). Since at the high temperatures we are considering the heating rate can be safely neglected, it follows that $t_{\text {cool }} \sim \rho^{-1}$. On the other hand, the free-fall time reads

$$
t_{f f} \sim(G \rho)^{-1 / 2}
$$

and so we see that $t_{\text {cool }}$ decreases faster than $t_{f f}$ as $\rho$ increases. As the above quasi-hydrostatic equilibrium state of the $\mathrm{PG}$ is characterized by the condition $t_{\text {cool }} \sim t_{f f}$, it is clear that inside the PGC clouds we have $t_{\text {cool }}<t_{f f}$. That is to say, the PGC clouds cool more rapidly than the rest of the PG. This process continues until hydrogen recombination occurs, because as soon as this happens - at a temperature $\sim 10^{4} \mathrm{~K}$ - the cooling rate decreases precipitously (under the equilibrium assumption) [52]. Therefore, the regime $t_{\text {cool }}>t_{f f}$ is now established even in the PGC clouds and so the PG can be regarded at this stage as a two-phase medium, with cold PGC clouds in pressure equilibrium with the external (inter PGC clouds) diffuse hot gas. 
However, Kang et al. [48] realized that the fast radiative cooling of the PGC clouds (from $10^{6}$ to $10^{4} \mathrm{~K}$ ) implies that the plasma inside these clouds cools more rapidly than it recombines, so that the above ionization equilibrium assumption is violated. Actually, the out-of-equilibrium recombination results in an enhanced ionization fraction. This fact does not affect the previous conclusions for temperatures $>10^{4} \mathrm{~K}$, but entails drastic changes at lower temperatures. Indeed, the existence of a sizable amount of protons and electrons at temperatures $<10^{4} \mathrm{~K}$ gives rise to $H_{2}$ formation via the following reactions ${ }^{4}$

$$
H+p \rightarrow H_{2}^{+}+\gamma, \quad H+e \rightarrow H^{-}+\gamma
$$

and

$$
H_{2}^{+}+H \rightarrow H_{2}+p, \quad H+H^{-} \rightarrow H_{2}+e
$$

As a consequence, the PGC clouds undergo a further cooling below $10^{4} \mathrm{~K}$. Specifically, there is a direct radiative cooling via reactions (4) and a radiative cooling via excitation of rotovibrational transitions of $\mathrm{H}_{2}$, produced by reactions (5). We stress that the latter process is very effective (much more than the former) at temperatures $<10^{4} \mathrm{~K}$ and plays a crucial rôle in our considerations. Since now $t_{\text {cool }} \ll t_{f f}$ in the PGC clouds, their density steadily rises. When the number density in the PGC clouds exceeds $10^{8} \mathrm{~cm}^{-3}$, the $H_{2}$ production increases dramatically thanks to the three-body reactions

$$
H+H+H \rightarrow H_{2}+H, \quad H+H+H_{2} \rightarrow H_{2}+H_{2}
$$

as pointed out by Palla et al. [50]. In effect, these reactions are so efficient that virtually all the atomic hydrogen gets rapidly converted ${ }^{5}$ to $H_{2}$. Therefore, the cooling of the PGC clouds is strongly enhanced and their evolution can proceed according to the scenario proposed by Palla et al. [50]: collapse and subsequent fragmentation of each PGC cloud into smaller clouds that remain optically thin until the minimum value of the Jeans mass $\left(\sim 10^{-2} M_{\odot}\right)$ is attained.

Still, it goes without saying that $H_{2}$ can be dissociated by various sources of ultraviolet (UV) radiation, like an active galactic nucleus (AGN) or a population of massive young

\footnotetext{
${ }^{4}$ We wish to emphasize that the familiar reaction $H+H \rightarrow H_{2}+\gamma$ (which requires grains in order to be efficient) is presently irrelevant, since no dust exists in the PGC clouds.

${ }^{5}$ Observe that, at variance with reactions (4) and (5), reactions (6) do not require the presence of electrons and protons as a catalyst.
} 
stars (population III) [53] at the centre of the PG. So, the ultimate fate of the PGC clouds strongly depends on the survival of $\mathrm{H}_{2}$.

As a matter of fact, in the early phase of the PG an AGN is expected to form at its centre along with population III stars, because of the disruption of central PGC clouds. This indeed happens, for the cloud collision time is shorter than the cooling time in the central region of the PG.

Thus, $H_{2}$ will be dissociated at galactocentric distances smaller than a certain critical value $R_{c r i t}$. Following the analysis of Kang et al. [48], it is straightforward to evaluate $R_{\text {crit }}$. Consider first the case of a UV flux due to a central AGN. Then we find

$$
R_{c r i t}^{A G N} \simeq\left(\frac{L_{A G N}}{2 \times 10^{42} \mathrm{erg} \mathrm{s}^{-1}}\right)^{1 / 2} \mathrm{kpc}
$$

For typical luminosities up to $L_{A G N} \sim 10^{45} \mathrm{erg} \mathrm{s}^{-1}$, eq.(7) yields $R_{\text {crit }}^{A G N} \sim 20 \mathrm{kpc}$. On the other hand, when the UV dissociating flux is produced by massive young stars mainly located at the centre of the PG, the critical galactocentric distance turns out to be

$$
R_{c r i t}^{\star} \simeq\left(\frac{10^{-3} \mathrm{kpc}^{-3}}{n_{0}}\right)\left(\frac{L_{t o t}}{L_{\star}}\right) \mathrm{kpc}
$$

In eq.(8) $L_{\star} \sim 2 \times 10^{38} \mathrm{erg} \mathrm{s}^{-1}$ is the bolometric luminosity of a single B0 V star. Assuming a total stellar luminosity $L_{\text {tot }}$ up to $\sim 2 \times 10^{45} \mathrm{erg} \mathrm{s}^{-1}$ and a central number density $n_{0}$ up to $\sim 10^{3} \mathrm{kpc}^{-3}$, we find $R_{c r i t}^{\star} \sim 10 \mathrm{kpc}$. In conclusion, $H_{2}$ should remain undissociated at galactocentric distances larger than $10-20 \mathrm{kpc}$.

\subsection{Globular clusters}

According to the foregoing analysis, in the inner galactic halo - that is for galactocentric distances smaller than $10-20 \mathrm{kpc}-H_{2}$ gets dissociated ${ }^{6}$, thus preventing any further cooling of the PGC clouds below $T \sim 10^{4} \mathrm{~K}$. Therefore, these clouds remain for a long time in quasi-hydrostatic equilibrium at $T \sim 10^{4} \mathrm{~K}$ (namely we have $t_{\text {cool }}>t_{f f}$ during this period). In such a situation a characteristic mass scale gets imprinted on the PGC clouds by gravitational instability, thereby resulting in a strongly peaked initial mass function. In fact, Fall and Ress [47] have shown that the Jeans mass of the PGC clouds after the long

\footnotetext{
${ }^{6}$ This indeed occurs for a wide range of UV fluxes and PGC cloud densities [48].
} 
permanence at $T \sim 10^{4} \mathrm{~K}$ is given (as a function of the galactocentric distance $R$ ) by ${ }^{7}$

$$
M_{P G C}(R) \simeq 5 \times 10^{5}(R / \mathrm{kpc})^{1 / 2} M_{\odot}
$$

while the $\mathrm{PGC}$ radius turns out to be

$$
r_{P G C}(R) \simeq 20(R / \mathrm{kpc})^{1 / 2} \mathrm{pc}
$$

Observe that in the present case the propagation of sound waves erases all large-scale perturbations, leaving only those at small scales.

Surely, this is not the end of the story, since the UV flux is expected to eventually decrease. So, after some time a sizable fraction of $H_{2}$ should anyway form, causing a sudden drop of the PGC cloud temperature well below $\sim 10^{4} \mathrm{~K}$ (the clouds now enter the regime $t_{\text {cool }}<t_{f f}$ ). What next happens is a rapid growth of the small-scale perturbations, which lead directly (in one step) - due to the thermal instability - to the formation of stars within a narrow mass range inside the PGC clouds [49]. In this way, globular clusters should form as they are observed today especially in the inner part of the galactic halo. Incidentally, the formation of the PGC clouds could have been delayed until after the Galaxy was enriched by metals due to population III stars, thus explaining the absence of globular clusters with primordial metal abundances and the radial gradient of metallicity in the galactic halo.

\subsection{Dark clusters of MACHOs and cold molecular clouds}

As we have seen, in the outer galactic halo - namely for galactocentric distances larger than $10-20 \mathrm{kpc}-\mathrm{H}_{2}$ molecules are not dissociated due to the absence of a sizable UV flux. This circumstance entails a very efficient cooling of the PGC clouds, whose state is therefore characterized by the condition $t_{c o o l}<t_{f f}$. Hence, the gravitational collapse can occur as in the scenario of Palla et al. [50], because of the very effective dissipation of gravitational energy by molecules and, owing to the decreasing Jeans mass for the internal gas, a PGC cloud can subsequently fragment into smaller clouds that remain optically thin to their own radiation. The process stops when the clouds become optically thick to their own emission. This happens when the Jeans mass is as low as $\sim 10^{-2} M_{\odot}$. We remark that the presence

\footnotetext{
${ }^{7}$ We stress that the $R$-dependence in eqs. (9) and (10) holds for $R \leq 20 \mathrm{kpc}$ only (see the discussion in Vietri and Pesce [54]). Indeed, as can be seen in Figure 5 in [54] the mass of globular clusters tends rather to decrease for $R \geq 20 \mathrm{kpc}$.
} 
of rotation and magnetic fields in the PGC clouds would delay the collapse process. As a result, this allows the Jeans mass to drop even further. Obviously, the fragmentation down to low-mass values is favoured if the initial gas has been metal enriched by population III stars (see Figure 7 in [55]).

The result of the above picture is the formation of dark clusters containing MACHOs, with masses in the range $10^{-2}-10^{-1} M_{\odot}$. As is well known, compact objects with these masses are brown dwarfs [56], which are not sufficiently massive to ignite hydrogen burning reactions ${ }^{8}$. We stress that - at variance with the case of globular clusters - the initial mass function of the dark clusters should be smooth, since the monotonic decrease of the PGC cloud temperature fails to single out any particular mass scale.

However, we don't expect the fragmentation process to be able to convert the whole gas in a PGC cloud into MACHOs. For instance, standard stellar formation mechanisms lead to an upper limit of at most $40 \%$ for the conversion efficiency [58]. Therefore, most of the remaining gas ought to form either diffuse or self-gravitating cold molecular clouds - mainly of $\mathrm{H}_{2}$ - which remain gravitationally bound in the dark clusters (since strong stellar winds are now absent). Moreover, we expect the mass ratio $f$ between the matter in the form of molecular clouds and the total matter in a dark cluster to be a function of the galactocentric distance $R$, depending on the environmental conditions, like incoming UV fluxes and collision rates among $\mathrm{PGC}$ clouds.

We would like to remark that the gas cannot have diffused in the whole galactic halo, for otherwise it would have been heated by the gravitational field to a virial temperature $\sim 10^{7}$ $\mathrm{K}$ and would therefore have been detected in the X-ray band (for which stringent upper limits are available [59]). The further possibility that the gas wholly collapsed into the disk is also excluded, because the disk mass would then be of the order of the inferred dark halo mass.

Whenever an important fraction of the gas is diffuse within a dark cluster, its temperature is given by the virial theorem as

$$
T \sim\left(\frac{M_{D C}}{M_{\odot}}\right)\left(\frac{\mathrm{pc}}{r_{D C}}\right) \mathrm{K}
$$

\footnotetext{
${ }^{8}$ Brown dwarfs have been discovered quite recently [57]. Notice that the lower limit $0.08 M_{\odot}$ for hydrogen burning gets slightly increased if the metallicity is lower than in the disk (private communication from $F$. D'Antona).
} 
with $r_{D C}$ and $M_{D C}$ denoting the dark cluster radius and mass, respectively. Hence, we see that we can easily run the risk to make the gas observable in optical and radio bands or even in soft X-rays. This problem disappears when the amount of gas is very low. This is actually the case in the scenario proposed by Gerhard and Silk [40], where dark cluster masses and dimensions are typically $M_{D C} \sim 10 M_{\odot}$ and $r_{D C} \sim 10 \mathrm{pc}$, so that the virial temperature is less than $10 \mathrm{~K}$. On the other hand, for a higher dark cluster mass, a way out of the above problem is provided by self-gravitating molecular clouds inside the dark cluster, since in this case the virial theorem has to hold for individual molecular clouds. Realistically, because of the negligible energy deposition from cosmic rays (see section 2.6), we expect the cloud temperature $T_{m}$ to be very close to that of the CBR (see section 3.2) and so the molecular cloud mass $M_{m}$ and radius $r_{m}$ are related via the virial theorem by

$$
M_{m} \sim 5\left(\frac{r_{m}}{\mathrm{pc}}\right) M_{\odot} .
$$

Assuming $10^{-3} M_{\odot}<M_{m}<10^{-1} M_{\odot}$, we get $10^{-3} \mathrm{pc}<r_{m}<10^{-1} \mathrm{pc}$ and number densities in the range $10^{4} \mathrm{~cm}^{-3}<n_{m}<10^{8} \mathrm{~cm}^{-3}$.

Equilibrium configurations of interstellar molecular clouds have been studied by several authors (see, e.g. $[60,61]$ ) by considering clouds in pressure equilibrium with their atomic envelopes and embedded in the galactic disk, where the ambient conditions (like radiation, dust and so on) are substantially different from those in the halo. In these models the gas is described by a barotropic equation of state $p \sim \rho^{\Gamma}$ with $\Gamma<1$, yielding a gas temperature which increases outwards, whereas the cloud pressure remains nearly constant. Such models could also apply to halo molecular clouds in the case of less massive dark clusters and the clouds could be stabilized against collapse by the MACHOs in the cluster, as discussed by Gerhard and Silk [40]. A different possibility for the equilibrium of the molecular clouds arises within models in which they are supported by self-gravity. These models are based on a polytropic equation of state $p \sim \rho^{\gamma}$ ( $\gamma$ being the usual ratio of specific heats). As is well known, the ensuing equilibrium configurations are stable provided $\gamma>4 / 3$, which is indeed our case since we are dealing with biatomic molecules.

It goes without saying that a more thorough analysis of these issues is required. 


\subsection{Dynamical constraints}

In order to play any rôle as a candidate for dark matter, dark clusters must have survived until today in the outer part of the galactic halo. This very fact sets highly nontrivial constraints on their masses and radii, as we are now going to discuss ${ }^{9}$.

We begin by assuming (as usual) that the halo dark matter density is modelled by an isothermal sphere, namely

$$
\rho(R)=\rho_{0} \frac{a^{2}+R_{G C}^{2}}{a^{2}+R^{2}}
$$

where $a \sim 5 \mathrm{kpc}$ is the halo core radius, $R_{G C} \sim 8.5 \mathrm{kpc}$ is our galactocentric distance and $\rho_{0}$ denotes the local dark matter density. It turns out that $\rho_{0}$ is directly related to the onedimensional velocity dispersion $\sigma$ of the halo. We shall take $\rho_{0}=0.65 \times 10^{-24} \mathrm{~g} \mathrm{~cm}^{-3}$, which corresponds to $\sigma=155 \mathrm{~km} \mathrm{~s}^{-1}$ (observe that the two-dimensional halo velocity dispersion is then $220 \mathrm{~km} \mathrm{~s}^{-1}$ ). Moreover, we shall denote by $\delta$ the fraction of halo dark matter in the form of dark clusters and in the considerations to follow we shall suppose for definiteness that the age of the Universe is $\sim 10^{10} \mathrm{yr}$ and that the average MACHO mass is $\sim 0.08 M_{\odot}$.

In spite of the fact that dark clusters form at galactocentric distances larger than 10-20 $\mathrm{kpc}$, they are subject to dynamical friction as they orbit through the galaxy, which makes them loose energy and therefore spiral in toward the galactic centre. However, it turns out that the ensuing drag is totally negligible as long as the dark cluster mass obeys the bound $M_{D C}<10^{7} M_{\odot}$, since the time that a dark cluster takes to reach the galactic centre is then definitely longer than the age of the Universe. This constraint also ensures that the dark clusters practically remain confined within the outer galactic halo. In particular, it follows that encounters between dark and globular clusters as well as disk shocking of dark clusters will be dynamically irrelevant.

Various effect may eventually lead to the disruption of dark clusters. We shall analyze them below.

As is well known, any stellar association evaporates in a finite time as a consequence of relaxation via star-star encounters. Clearly, the larger the cluster size, the longer the lifetime. So, the request that dark clusters still exist today sets a lower limit on their radius. Specifically, we find $r_{D C}>10.2 \times\left(M_{\odot} / M_{D C}\right)^{1 / 3} \mathrm{pc}$.

A different question concerns encounters between dark clusters, which will be considered

\footnotetext{
${ }^{9} \mathrm{~A}$ more detailed treatment of this point will be reported elsewhere.
} 
below in the impulse approximation (i.e. for relative velocities between dark clusters much higher than the internal velocity dispersion). Let us analyze first distant encounters. Then, the typical time $t_{d}$ needed by these encounters to dissolve a dark cluster can be straightforwardly computed following Binney and Tremaine [62]. Requiring next $t_{d}$ to exceed the age of the Universe, we find $r_{D C}<(14.5 / \delta)(R / 10 \mathrm{kpc})^{2}$ pc. Penetrating encounters can be handled in a similar fashion. This task is most simply accomplished by modelling the clusters by means of a Plummer potential with core radius $b$. As before, the ensuing disruption time $t_{d}$ has to exceed the age of the Universe if the dark clusters have to survive until today. Taking $b \simeq 0.1 \times r_{D C}$, we get $r_{D C}<(1.3 / \delta)(R / 10 \mathrm{kpc})^{2}$ pc. As expected, the latter bound is stronger than the former and both of them are independent of $M_{D C}$.

Finally, we briefly address the disruption of dark clusters by tidal perturbations from the galactic gravitational field. Indeed, if the dark cluster radius is "too large", self-gravity starts to compete with the gravitational field of the Galaxy: in this manner, the binding energy of MACHOs and molecular clouds decreases and the cluster can eventually evaporate. Accordingly, we get $r_{D C}<1.4 \times\left(M_{D C} / M_{\odot}\right)^{1 / 3}(R / 10 \mathrm{kpc})^{2 / 3} \mathrm{pc}$.

As it is clear, all these constraints can be fulfilled for a wide range of dark cluster masses and radii. Notice, however, that all dark clusters should be less massive than $10^{7} M_{\odot}$.

\subsection{Molecular clouds: composition}

Besides $H_{2}$, molecules made of primordial elements such as $H D$ and $L i H$ can be present in the inner region of the molecular clouds, provided the temperature is low enough and the self-shielding is effective. Moreover, also molecules made of metals can be present in the halo clouds, even if in lower amount than in globular clusters. As far as self-shielding is concerned, we notice that a $H_{2}$ column density of $\sim 10^{14} \mathrm{~cm}^{-2}$ is sufficient to shield disk clouds from the external UV flux [63]. The corresponding layer in the halo molecular clouds is less than $\sim 10^{-6} \mathrm{pc}$ in thickness, which should be viewed as an upper limit, since we expect the UV flux to decrease at large galactocentric distances.

In the following, we provide an estimate of the halo cloud composition which should, however, merely be taken as an indication. To this purpose, we start from the observed molecular abundance of interstellar clouds like $\zeta$ Ophiuchi (given by Viala et al. [64]). These abundances are also confirmed by comprehensive models for interstellar molecular clouds 
with solar composition $[65,60]$. We use these informations to evaluate the final molecular abundances for halo clouds, assuming however a lower initial metallicity and temperature. In fact, column $A$ of Table 5 in Millar et al. [65] yields the molecular fractional abundances for a cloud at $T=10 \mathrm{~K}$ and initial solar composition after $3.16 \times 10^{5} \mathrm{yrs}$ from its formation. At this early time the molecular abundance $n_{A B}$ of the species $A B$ increases linearly as $n_{A B} \sim n_{A} n_{B}<\sigma v>t$. Here $n_{A}$ and $n_{B}$ are the number density of atoms $A$ and $B, \sigma$ is the cross-section for molecule formation, $v$ is the relative velocity of the atoms and $t$ is the time. Thus, a simple convolution that takes into account the smaller relative velocity distribution of the atoms (corresponding to a cloud temperature $T_{m} \sim 3 \mathrm{~K}$ - see section 3.2) and a lower initial metallicity (rescaled down by a factor up to $10^{-4}$ with respect to the solar one) allows us to estimate the present abundances (after $\sim 10^{10}$ yrs from the cloud formation) for the molecules considered in the Table. As we shall see in section 3.3, these abundances have to be regarded as lower limits in order to have a chance to detect halo molecular clouds through high-sensitivity spectroscopy towards stars in the LMC.

\subsection{Molecular clouds: thermal balance}

We proceed to estimate the temperature of halo molecular clouds by studying their thermal balance. To this end, we need to know the heating rate $\Gamma$ (due to external sources) and the cooling rate $\Lambda$ (due to the molecules). In the galactic halo, the dominant heat source for molecular clouds is expected to be ionization from photons of the $\mathrm{X}$-ray background, whose spectrum in the relevant range $1 \mathrm{keV}<E<25 \mathrm{keV}$ (see below) can be parameterized in terms of the energy $E$ (expressed in $\mathrm{keV}$ ) as $I(E)=8.5 E^{-0.4} \mathrm{~cm}^{-2} \mathrm{~s}^{-1} \mathrm{sr}^{-1}$ [66]. The ionization rate for $\mathrm{H}_{2}$ molecules (taking into account secondary ionization) is

$$
\xi_{X}=26 \int_{E_{\min }}^{E_{\max }} 4 \pi I(E) \sigma_{X}(E) d E \mathrm{~s}^{-1},
$$

where $\sigma_{X}(E)=2.6 \times 10^{-22} E^{-8 / 3} \mathrm{~cm}^{2}$ is the absorption cross-section in the above energy range for incoming $X$-rays on gas with interstellar composition [67]. Actually, this expression for $\sigma_{X}(E)$ has to be considered as an upper limit, since we expect that for molecular clouds with a lower metallicity (with respect to the solar one) the energy dependence of $\sigma_{X}(E)$ should decrease (see Fig.1 in [67]). The integration limits $E_{\min }$ (below which X-rays are absorbed) and $E_{\max }$ (above which X-rays go through the whole cloud without being absorbed) depend on the cloud column density. Thus, if we suppose that $n_{H_{2}} \sim n_{m}$ is in the range 
$10^{4} \mathrm{~cm}^{-3}<n_{H_{2}}<10^{8} \mathrm{~cm}^{-3}$, as it follows from section 2.3 , we get $1.25 \mathrm{keV}<E_{\min }<7 \mathrm{keV}$ and $10 \mathrm{keV}<E_{\max }<20 \mathrm{keV}$. It turns out that $\xi_{X}$ does not depend much on the upper limit. Therefore, we obtain $\xi_{X}=2.2 \times 10^{-19} \mathrm{~s}^{-1}$ for $E_{\min }=1.25 \mathrm{keV}$ and $\xi_{X}=5.6 \times 10^{-21} \mathrm{~s}^{-1}$ for $E_{\text {min }}=7 \mathrm{keV}^{10}$. Since each ionization releases an energy of $\sim 8 \mathrm{eV}$, the heating rate per $H_{2}$ molecule $\tilde{\Gamma}$ turns out to be

$$
3.5 \times 10^{-32} \frac{\mathrm{ergs}}{\mathrm{s} H_{2}}<\tilde{\Gamma}<1.5 \times 10^{-30} \frac{\mathrm{ergs}}{\mathrm{s} H_{2}} .
$$

Unfortunately, the cooling function for the halo clouds in question is not well known, owing to the lack of information on their chemical composition. Nevertheless, by considering only the cooling rate due to $\mathrm{H}_{2}$ as given by Goldsmith and Langer [68], the equality between cooling and heating rate per molecule leads to cloud temperatures $\sim 10-30 \mathrm{~K}$. More complete models for the cooling rate, which include the contribution from $H D$ and heavy molecules, imply that the cooling efficiency is substantially enhanced [68] and thus make it very plausible that halo molecular clouds clumped in dark clusters should have a temperature close to that of the CBR (see also Gerhard and Silk [40] for similar conclusions).

Manifestly, a more detailed analysis of this issue is needed.

\section{Observational Tests}

Let us now address the signatures of the above scenario, in addition to the individual MACHO detection via microlensing observations towards the LMC. The most promising way to decide whether MACHOs are clumped into dark clusters is through correlation effects in microlensing observations. For the more massive dark clusters in the range $10^{5}-10^{6} M_{\odot}$, the implied degeneracy in the spatial and velocity distributions would result in a strong autocorrelation in the sky position of microlensing events on an angular scale of $20^{\prime \prime}$, along with a correlation in their duration. It has been pointed out that already a fairly poor statistics would be sufficient to rule out the possibility that MACHOs are clumped into such massive clusters, while to confirm it more events are needed [69]. On the other hand, if either the mass of the dark clusters is much smaller than $10^{6} M_{\odot}$ or the fraction of dark matter in the form of MACHOs is small, then a much larger sample of events may be required.

\footnotetext{
${ }^{10} \mathrm{It}$ is straightforward to verify that the ionization due to the halo cosmic rays (which are relevant for the production of $\gamma$-rays through inelastic high-energy pp collisions) is less important in this context. In fact, assuming the ionization rate typical for disk cosmic rays $\xi_{0}=5 \times 10^{-17} \mathrm{~s}^{-1}[60]$ and rescaling for the diffusion of the cosmic rays in the galactic halo (see section 3.1), we infer $\xi_{c r} \sim 10^{-23} \mathrm{~s}^{-1}$.
} 


\section{1 $\gamma$-ray flux produced by halo molecular clouds}

We proceed to estimate the $\gamma$-ray flux produced in molecular clouds inside a dark cluster through the interaction with high-energy cosmic-ray protons. Cosmic rays scatter on protons in the molecules producing $\pi^{0}$ 's, which subsequently decay into $\gamma^{\prime}$ s. Outside the clouds we expect negligible high-energy $(\geq 100 \mathrm{MeV}) \gamma$-ray photon absorption in the interstellar medium, since for the typical interstellar medium density of $\sim 1$ atom $\mathrm{cm}^{-3}$ the mean free path is $\sim 20 \mathrm{Mpc}$.

An essential ingredient is the knowledge of the cosmic-ray flux in the halo. Unfortunately, this quantity is experimentally unknown and the only available information comes from theoretical estimates (see e.g. [70]). More precisely, from the mass-loss rate of a typical galaxy, we infer a total cosmic-ray flux in the halo $F \simeq 10^{41} A_{g a l}^{-1} \mathrm{erg} \mathrm{cm}^{-2} \mathrm{~s}^{-1}$, where $A_{\text {gal }}$ is the surface area of the galactic disk. Taking $R_{g a l} \simeq 10 \mathrm{kpc}$, we get

$$
F \simeq 3.5 \times 10^{-5} \frac{\mathrm{erg}}{\mathrm{cm}^{2} \mathrm{~s}}
$$

However, this information is not sufficient to carry out our calculations, since we need to know the energy distribution of the cosmic rays, for which we assume the same energy dependence as measured on the Earth. Then we rescale the overall density in such a way that the integrated energy flux agrees with the above value. Moreover, we suppose that the cosmic-ray density scales with the galactocentric distance $R$ as the dark matter density (see eq.(13)). Now, the measured primary cosmic-ray flux on the Earth is (see e.g. [71])

$$
\Phi_{C R}^{\oplus}(E) \simeq \frac{2}{\mathrm{GeV}}\left(\frac{E}{\mathrm{GeV}}\right)^{-2.7} \frac{\text { particles }}{\mathrm{cm}^{2} \mathrm{~s} \mathrm{sr}} .
$$

Therefore, the corresponding integrated energy flux is

$$
\int d \Omega d E E \Phi_{C R}^{\oplus}(E) \simeq 5.7 \times 10^{-2} \frac{\mathrm{erg}}{\mathrm{cm}^{2} \mathrm{~s}}
$$

where for definiteness we take the integration range to be $1 \mathrm{GeV} \leq E \leq 10^{6} \mathrm{GeV}$. Hence, the comparison between eqs. (16) and (18) entails $\Phi_{C R}(E) \simeq 6 \times 10^{-4} \Phi_{C R}^{\oplus}$, so that from eq.(17) we have

$$
\Phi_{C R}(E) \simeq \frac{1.2 \times 10^{-3}}{\mathrm{GeV}}\left(\frac{E}{\mathrm{GeV}}\right)^{-2.7} \frac{\text { particles }}{\mathrm{cm}^{2} \mathrm{~s} \mathrm{sr}},
$$

and - in accordance with our previous assumption - we finally obtain

$$
\Phi_{C R}(E, R) \simeq \Phi_{C R}(E)\left(\frac{a^{2}+R_{G C}^{2}}{a^{2}+R^{2}}\right)
$$


We stress that our result exhibits only a very mild $a$-dependence.

Let us now turn our attention to the evaluation of the $\gamma$-ray flux produced through the reactions $p p \rightarrow \pi^{0} \rightarrow \gamma \gamma$. Accordingly, the source function $q_{\gamma}(r)$, which gives the photon number density at distance $r$ from the Earth, is [72]

$$
q_{\gamma}(r)=\frac{4 \pi}{m_{p}} \sum_{n} \int d E_{p} d E_{\pi} \Phi_{C R}\left(E_{p}, R(r)\right) \rho_{H_{2}}(R(r)) \frac{d \sigma_{p \rightarrow \pi}^{n}\left(E_{\pi}\right)}{d E_{\pi}} n_{\gamma}\left(E_{p}\right)
$$

where $\sigma_{p \rightarrow \pi}^{n}\left(E_{\pi}\right)$ is the cross-section for the reaction $p p \rightarrow n \pi^{0}$ ( $n$ is the $\pi^{0}$ multiplicity), $\rho_{H_{2}}(R(r))$ is the molecular density, $n_{\gamma}\left(E_{p}\right)$ is the photon multiplicity [73]

$$
n_{\gamma}\left(E_{p}\right) \simeq 2+1.03 n_{c h}\left(E_{p}\right)
$$

while $R(r)$ is the galactocentric distance as a function of $r$ (see eq.(25) below). In order to proceed further, it is convenient to re-express $q_{\gamma}(r)$ in terms of the inelastic pion production cross-section $\sigma_{i n}\left(p_{l a b}\right)$. Since

$$
\sigma_{i n}\left(p_{l a b}\right)<n_{\gamma}\left(E_{p}\right)>=\sum_{n} \int d E_{\pi} \frac{d \sigma_{p \rightarrow \pi}^{n}\left(E_{\pi}\right)}{d E_{\pi}} n_{\gamma}\left(E_{p}\right)
$$

eq.(21) becomes

$$
q_{\gamma}(r)=\frac{4 \pi}{m_{p}} \rho_{H_{2}}(R(r)) \int d E_{p} \Phi_{C R}\left(E_{p}, R(r)\right) \sigma_{i n}\left(p_{l a b}\right)<n_{\gamma}\left(E_{p}\right)>.
$$

Actually, the cosmic-ray protons in the halo which originate from the galactic disk are mainly directed outwards. This circumstance implies that the induced photons will predominantly leave the Galaxy. However, the presence of magnetic fields in the halo might give rise to a temporary confinement of the cosmic rays similarly to what happens in the disk [74]. In addition, there could also be sources of cosmic rays located in the halo itself, as for instance isolated or binary pulsars in globular clusters. As we are unable to give a quantitative estimate of the above effects, we take them into account by introducing an efficiency factor $\epsilon$, which could be rather small. In this way, the $\gamma$-ray photon flux reaching the Earth is obtained by multiplying $q_{\gamma}(r)$ by $\epsilon / 4 \pi r^{2}$ and integrating the resulting quantity over the cloud volume along the line of sight. Let us parameterize a generic point $\mathrm{P}$ in the cloud volume by polar coordinates $(r, \theta, \phi)$, taking the Earth as the origin. Then, denoting by $R$ the galactocentric distance of $P$, we get

$$
R(r)=\left(r^{2}+R_{G C}^{2}-2 r R_{G C} \cos \theta \cos \phi\right)^{1 / 2}
$$


Because $d V=r^{2} d r d \Omega$, it follows that the observed $\gamma$-ray flux per unit solid angle is

$$
\Phi_{\gamma}(\theta, \phi)=\frac{\epsilon}{4 \pi} \int_{r_{1}}^{r_{2}} d r q_{\gamma}(r),
$$

where typical values of $r_{1}$ and $r_{2}$ are $10 \mathrm{kpc}$ and $50 \mathrm{kpc}$, respectively. So, we find

$$
\Phi_{\gamma}(\theta, \phi)=\epsilon f \frac{\rho_{0}}{m_{p}} I_{1}(\theta, \phi) I_{2}
$$

where $I_{1}(\theta, \phi)$ and $I_{2}$ are given by

$$
\begin{gathered}
I_{1}(\theta, \phi)=\int_{r_{1}}^{r_{2}} d r\left(\frac{a^{2}+R_{G C}^{2}}{a^{2}+R^{2}}\right)^{2}, \\
I_{2}=\int_{1 \mathrm{GeV}}^{10^{6} \mathrm{GeV}} d E_{p} \Phi_{C R}\left(E_{p}\right) \sigma_{i n}\left(p_{l a b}\right)<n_{\gamma}\left(E_{p}\right)>
\end{gathered}
$$

and we have assumed for $\rho_{H_{2}}(R(r))$ the same $R(r)$-dependence as in eq.(13). Accordingly, we obtain

$$
I_{1}\left(90^{\circ}\right) \simeq 10^{22} \mathrm{~cm} .
$$

Next, we focus our attention to the evaluation of $I_{2}$. We achieve this goal by using $\sigma_{i n}\left(p_{l a b}\right)$ as given in Particle Data Group [75], by re-expressing the integrand in terms of the Mandelstam variable $s$ (hence the integration range is presently $4 \mathrm{GeV}^{2} \leq s \leq 2 \times 10^{6} \mathrm{GeV}^{2}$ ) and by adopting in eq.(22) the following expression of the charged-particle multiplicity [75]

$$
<n_{c h}(s)>\simeq-7.4+7.6 s^{0.124} \text {. }
$$

Collecting everything together, $I_{2}$ turns out to be

$$
I_{2} \simeq 3.8 \times 10^{-28} \frac{\text { photons }}{\mathrm{s} \mathrm{sr}} .
$$

The best chance to detect the $\gamma$-rays in question is provided by observations at high galactic latitude. Therefore we set $\theta=90^{\circ}$ in eq.(27), thus finding

$$
\Phi_{\gamma}\left(90^{0}\right) \simeq \epsilon f 1.1 \times 10^{-6} \frac{\text { photons }}{\mathrm{cm}^{2} \mathrm{~s} \mathrm{sr}} .
$$

We should note that, although we have been concerned with the $\gamma$-ray production due to $p p$ collisions in molecular clouds, the same effect occurs for atomic hydrogen clouds as well. Clearly, a possibility to discriminate between the two components consists in performing a 
simultaneous search for the $21-\mathrm{cm}$ line, from which the atomic hydrogen abundance can be deduced [76] (and subtracted).

The inferred upper bound for $\gamma$-rays in the $0.8-6 \mathrm{GeV}$ range at high galactic latitude is $3 \times 10^{-7}$ photons $\mathrm{cm}^{-2} \mathrm{~s}^{-1} \mathrm{sr}^{-1}$ [77]. Hence, we see from eq.(33) that the presence of halo molecular clouds does not lead nowadays to any contradiction with such an upper limit, provided $\epsilon f<10^{-1}$. An improvement of sensitivity for the next generation of satellite-borne $\gamma$-ray detectors would either discover the effect in question or yield more stringent limits on $\epsilon f$.

An attempt to estimate $\epsilon$ and consequently the amount of dark diffuse gas has been recently done by Salati et al. [78]. They consider a model of cosmic rays which correctly reproduces their diffusion inside the galactic disk. By using recent EGRET data on diffuse $\gamma$-rays, a stringent limit on the abundance of dark baryonic gas $(\leq 4 \%)$ has been obtained in the inner galactic halo (for heights over the plane less than $\sim 4 \mathrm{kpc}$ ). No limits can be set on the scenario under consideration, in which dark clusters form at a distance larger than $10-20 \mathrm{kpc}$ in the galactic halo ${ }^{11}$.

\subsection{Cosmic Background Radiation Anisotropy}

Remarkably enough, molecular clouds clumped into dark clusters can be detected via the anisotropy they would introduce in the CBR owing to their nontrivial interaction [79].

Surely, the considered effect shows up provided the present temperature $T_{m}$ of the molecular clouds exceeds that of the CBR. In order to be definite, we assume that all dark clusters are spherically distributed at $\sim 20 \mathrm{kpc}$ from the galactic centre. Taking $r_{D C} \sim 10 \mathrm{pc}$, the angular size of a dark cluster is $\sim 1.8^{\prime}$. Hence, we expect to see about a dozen of dark clusters per square degree (provided observations are made away from the galactic center and off the galactic disk). An upper limit on $T_{m}$ can be obtained by supposing that the clouds are optically thick at every frequency. Accordingly, on account of the low surface filling factor $S \sim 10^{-2}$ ( $S$ is defined as the ratio of filled to total surface in a field of view), the ratio of the temperature excess of the clouds to the CBR temperature turns out to be less than $\sim 10^{-3}$. This bound can further be strengthened through observational limits on the anisotropy of the CBR on angular scales of $\sim 10^{\prime}$ [80], thanks to the corresponding increase of $S$.

\footnotetext{
${ }^{11}$ As we have seen in section 2.4, in spite of dynamical friction dark clusters remain confined within the outer galactic halo, where - according to Salati et al. [78] - the diffusion of cosmic rays vanishes.
} 
However, it should be remarked that in reality molecular clouds are not optically thick at every frequency and therefore they cannot be regarded as a black body. In fact, a molecular cloud close to the CBR temperature emits a set of lines due to molecular rotational transitions, thus the above limit can be softened.

Now, as long as the clouds have a cosmological primordial composition, the only molecule that contributes to the microwave band with optically thick lines is $\mathrm{LiH}$. This conclusion can be reached as follows. We take a cosmological abundance $n_{L i H} / n_{H_{2}} \sim 10^{-10}$. The resonance cross-section (at $T=2.76 \mathrm{~K}$ ) corresponding to the lowest-lying rotational transition of $\mathrm{LiH}$ at the frequency $\nu_{0}=444 \mathrm{GHz}$ is $\sigma_{\nu} \sim 5 \times 10^{-10} \mathrm{~cm}^{2}$ [81] and the associated optical depth reads

$$
\tau_{\nu}=\int \sigma_{\nu} n_{L i H} d l
$$

where the integral has to be performed along the line of sight intercepted by the molecular clouds. We find $\tau_{\nu}>1$, which shows that the line under consideration is indeed optically thick (this conclusion has been verified for the range of molecular cloud masses and densities given in section 2.3). Still, the precise chemical composition of the molecular clouds in the galactic halo is uncertain (see section 2.5). For instance, even if the $C O$ molecule abundance is very low (as compared with the abundance in interstellar clouds), the lines corresponding to the lowest-lying rotational transitions in the microwave band are optically thick. The same could well happen for other molecules.

Consider now molecular clouds in M31, for which we adopt the same scenario outlined above for our galaxy. Our distance from M31 is $D \sim 650 \mathrm{kpc}$. Although it is not clear what is the rotational velocity of the M31 halo (and also the radial anisotropy degree of the velocity distribution), we expect dark clusters to have typical rotational velocities of $50-100 \mathrm{~km}$ $\mathrm{s}^{-1}$.

As discussed above, molecular clouds are at a temperature very close to that of the CBR: this fact would make them indistinguishable from the background. But - since the dark clusters containing the clouds are moving - there is a Doppler shift. At worst, we expect at least the lowest rotational transition line of $\mathrm{LiH}$ at $\nu_{0}=444 \mathrm{GHz}$ to be present. For this purpose, it is convenient to perform narrow-band measurements, since the rotational velocity of the dark clusters causes a Doppler shift $\Delta \nu / \nu_{0} \sim 3 \times 10^{-4}$ (the line broadening, mainly due to the turbulent velocity of the molecular clouds inside a dark cluster, is anyway 
smaller than this).

On the other hand, if many lines fall in the microwave band, it could be preferable to perform broad-band measurements, which, while diluting the effect, nevertheless have a higher sensitivity. In the latter case, the Doppler shift effect will show up as an anisotropy in the CBR. This phenomenon is the Doppler shift for a free-streaming gas with speed $v$ [82]. The corresponding anisotropy is then

$$
\frac{\Delta T}{T}= \pm \frac{v}{c} S f \tau_{\nu}
$$

The sign depends on the direction of the projected velocity along the line of sight and $T$ is the temperature of the CBR. The optical depth $\tau_{\nu}$ is a function of the molecular abundance, the number of molecules in each quantum level and the resonance cross-section (see [83] for details). Because the clouds are optically thick only at some frequencies, it is better to use in eq.(35), instead of $\tau_{\nu}$, the average optical depth over the frequency range $\left(\nu_{1} \leq \nu \leq \nu_{2}\right)$ of the detector

$$
\bar{\tau}=\left(\nu_{2}-\nu_{1}\right)^{-1} \int_{\nu_{1}}^{\nu_{2}} \tau_{\nu} d \nu
$$

So, if we could look for anisotropies in the CBR towards M31 with resolution of $\sim 3^{\prime \prime}$, eq.(35) would imply anisotropies of $\sim 3 \times 10^{-4} f \bar{\tau}$ in $\Delta T / T$ (since $S \sim 1$ ).

As it is difficult to work with fields of view of a few arcsec, we calculate below the expected CBR anisotropy between two fields of view (on opposite sides of M31) separated by $\sim 4^{0}$ and with angular resolution of $\sim 1^{0}$. Supposing that the halo of M31 consists of $\sim 10^{6}$ dark clusters and that all of them lie between $25 \mathrm{kpc}$ and $35 \mathrm{kpc}$, we would be able to detect $10^{3}-10^{4}$ dark clusters per square degree. Scanning an annulus of $1^{0}$ width and internal angular diameter $4^{\circ}$ (see Figure), centered at M31, in 180 steps of $1^{0}$, we would find anisotropies of $\sim 2 \times 10^{-5} f \bar{\tau}$ in $\Delta T / T$ (as now $S \sim 1 / 25$ ). In conclusion, the theory does not permit to establish whether the expected anisotropy lies above or below current detectability $\left(\sim 10^{-6}\right)$, and so only observations can resolve this issue.

Incidentally, we should point out that broad-band measurements can be very difficult due to the contribution from cirruses emission in the microwave band. Cirruses in M31 itself should not interfere with the above-proposed measurements of the CBR anisotropies, since they lie inside $\sim 15 \mathrm{kpc}$, as it follows from IRAS observations [84]. Another problem are foreground cirruses within our galaxy located in the direction of M31, that IRAS showed 
to be inhomogeneously distributed [85]. Their contribution could be subtracted using the available IRAS maps. On the other hand, it is well possible that there exists cold dust, whose emission results in a spectrum that peaks beyond the longest wavelength IRAS band $(100 \mu \mathrm{m})$ and whose presence could be inferred by performing the above-considered measurements in the microwave band with different decreasing angular widths rather than using a fixed width of $1^{0}$. Accordingly, since the filling factor does not change, the presence of cold dust along the field of view shows up as constant values for the CBR anisotropy. Otherwise, in the absence of foreground cold dust, we should find an increase in the $\Delta T / T$ values.

\subsection{Absorption lines}

An attractive strategy to discover the halo molecular clouds clumped into dark clusters relies upon the absorption lines they would introduce in the spectrum of a LMC star [86].

Consider a microlensing event towards the LMC due to a MACHO which, for the sake of definiteness, we suppose to be located inside the central region of a dark cluster with $M_{D C} \sim 10^{6} M_{\odot}$ and $r_{D C} \sim 10 \mathrm{pc}$. As we have seen, the angular size of such a cluster is $\sim 1.8^{\prime}$ for a typical distance of $\sim 20 \mathrm{kpc}$. Assuming indicatively $M_{m} \sim 10^{-1} M_{\odot}$, the number of clouds per cluster turns out to be $\sim 10^{7} \mathrm{f}$. In addition, the virial theorem entails that $r_{m} \sim 2 \times 10^{-2} \mathrm{pc}$, and so the mean number density of the clouds in question is $n_{m} \sim 10^{5} \mathrm{~cm}^{-3}$. Therefore, the light of the LMC stars within a field of angular size $\sim 0.9^{\prime}$ centered at the microlensed star will go through $\sim 10^{2} f$ molecular clouds of the dark cluster under consideration.

We adopt the cloud composition discussed in section 2.5. Nevertheless, we would like to emphasize that if the formation of molecules proceeds in a different fashion, our conclusions can still hold true. In fact, as long as one of the molecules listed in the Table has an abundance equal to or larger than the one reported in the Table, the corresponding lines will be detectable. Thus, a measurement as proposed below would also be interesting for the determination of the molecular content of the galactic halo.

In spite of the very low abundance, some molecules do have optically thick absorption lines in the UV and optical bands, because of the dramatic enhancement of the resonance crosssection. Hence, these lines will show up in the spectrum of stars within the above-mentioned field. Moreover, here we assume an instrumental spectral resolution $\Delta \nu / \nu \sim 10^{-5}$, which is 
within the present technological capabilities (see e.g. Pasquini et al. [87] and Brandt [88]) and should become feasible with a next generation of instruments.

Since we are concerned with high resolution spectroscopy, we shall focus our attention on electronic molecular transitions falling in the optical and UV bands ${ }^{12}$. We expect optically thick lines in molecules with high permanent electric dipole moment. Here, as an illustration, we consider the molecules $\mathrm{LiH}, \mathrm{CO}, \mathrm{CH}, \mathrm{CN}$, whose fractional abundance with respect to $\mathrm{H}_{2}$ is exhibited in the Table together with wavelengths and classification of some radiation lines.

We now present an estimate of the molecular abundances necessary to make the lines optically thick, that is to say detectable. We adopt the narrow-optical-line approximation [89], since for a cloud temperature $T_{m} \sim 3 \mathrm{~K}$ the natural line width is $\Delta \nu / \nu=2(\ln 2)^{1 / 2} b / c \sim$ $6 \times 10^{-7}$, with $b=\left(2 k T_{m} / m\right)^{1 / 2} \sim 10^{4} \mathrm{~cm} \mathrm{~s}^{-1}$. Therefore, the equivalent line width is given by $W_{\lambda} / \lambda=2 b F\left(\tau_{0}\right) / c$, where the curve of growth $F\left(\tau_{0}\right)$ is tabulated (see Table 3.1 in Spitzer [89]) as a function of the central optical depth $\tau_{0}=1.497 \times 10^{-2}<n_{m}>l_{c} \lambda_{j k} f_{j k} / b$ for a Maxwellian velocity distribution. Here, $\left\langle n_{m}\right\rangle$ is the mean number density of the considered molecule and $l_{c} \sim 10^{-2} \mathrm{pc}$ is the cloud size along the line of sight.

Aa we have seen, the light coming from a LMC star typically intercepts $\sim 10^{2} f$ molecular clouds and each of them has dispersion velocity $\sim 10 \mathrm{~km} \mathrm{~s}^{-1}$ within the dark cluster (taking as above $M_{D C} \sim 10^{6} M_{\odot}$ and $r_{D C} \sim 10 \mathrm{pc}$ ). Correspondingly, due to the Doppler shift effect, we expect $\sim 10^{2} f$ lines around the considered transition frequency owing to the velocity dispersion of the clouds. As a consequence, the overall equivalent line width becomes $\sim 200 f b F\left(\tau_{0}\right) / c$. Assuming indicatively $f \sim 0.5$, in order to have an absorption feature detectable with a $10^{-5}$ spectral resolution instrument, we need $F\left(\tau_{0}\right) \sim 0.3$, implying a minimum molecular abundance $\left\langle n_{m}>_{\min } \sim 5 \times 10^{-12}\left(\lambda_{j k} f_{j k}\right)^{-1} \mathrm{~cm}^{-3}\right.$. For example, in the case of the $C O$ molecule this yields $n_{C O} / n_{H_{2}} \leq 10^{-9}$, which is the value quoted in the Table.

We remark that we do not expect the presence of a sizable amount of dust in the halo clouds, because grains typically form in stellar atmospheres where high temperatures are established. In any case, if the dust to gas mass ratio is such that $M_{d u s t} / M_{\text {gas }} \leq 10^{-5}$ (which is $\sim 10^{-3}$ less than the corresponding ratio of about $10^{-2}$ for interstellar disk clouds [89]) the dust optical depth remains at all wavelengths well below one and so a background source

\footnotetext{
${ }^{12}$ Actually, also electronic transitions of atoms possibly present in the molecular clouds can manifest themselves as absorption lines and should be taken into account in a more thorough analysis.
} 
cannot be obscured ${ }^{13}$.

Some comments are in order. i) Bright $\mathrm{O}$ and $\mathrm{B}$ stars are the most reliable candidates to search for absorption lines, thanks to the absence of intrinsic lines. Unfortunately, the probability to find such a star in a field of view of $\sim 1^{\prime}$ (the angular radius of a dark cluster) is $\sim 10^{-2}$. This estimate comes from the existence of at least $10^{3}$ luminous $\mathrm{O} / \mathrm{B}$ stars in the LMC [91] as well as from the determination of the LMC stellar initial mass function (see also [92]). The probability to find a luminous star in a field of view of $1^{\prime}$ around a previously microlensed one increases by about one order of magnitude if a microlensing event is found towards young stellar associations in the LMC (such as 30 Doradus), where a high concentration of luminous stars is present. Regretfully, present microlensing experiments use large exposure times to observe low-luminosity stars (which are more abundant) and do not operate towards 30 Doradus in order to prevent camera saturation. At any rate, to have an appreciable chance to find an $\mathrm{O} / \mathrm{B}$ star near a previously microlensed one it requires an increase of at least one order of magnitude in the microlensing detection rate. This sensitivity can well be reached within the next generation of planned microlensing searches, like EROS II and OGLE II which are both scheduled to start during 1996. Moreover, since the apparent position of the halo dark clusters relative to the LMC stars does not change significantly over a period of several years, one may reasonably hope to accumulate $\sim 100$ or more events within some years of continuous monitoring for microlensing. Alternatively, late-type stars can be contemplated. Accordingly, in order to distinguish between the intrinsic features of such stars and the absorption lines induced by the intercepted molecular clouds, one should compare absorption spectra of identical spectral-type stars located inside and outside $\sim 1^{\prime}$ around a previously microlensed one. ii) A method to discriminate among Milky Way, LMC, Magellanic Stream gas (possibly intercepted by the line of sight) and the halo clouds in question is needed. Foreground clouds are fundamentally different with respect to the halo molecular clouds, owing to the presence of grains and their consequent higher temperature (that leads to broader absorption lines). Then IRAS maps (in which the grain emission shows up) as well as careful measurements in different bands of the electromagnetic spectrum can

\footnotetext{
${ }^{13}$ Here, we adopted the standard extinction curve for the observed disk clouds, according to which at the wavelength $\sim 0.1 \mu \mathrm{m}$ the extinction cross-section is $\tau_{e x t}=2.3 \times 10^{-21}\left(N_{H} / \mathrm{cm}^{-2}\right)$, where $N_{H}$ denotes the hydrogen column density (see Fig. 7 in [90]). This means that for the considered halo clouds we have $\tau_{\text {ext }}=1.5 f\left(n_{H_{2}} / \mathrm{cm}^{-3}\right)\left(M_{\text {dust }} / M_{\text {gas }}\right)$ for $\sim 10^{2} f$ intercepted molecular clouds along the line of sight. Therefore, in order that $\tau_{\text {ext }} \ll 1$ we need $M_{\text {dust }} / M_{\text {gas }} \leq 10^{-5}$.
} 
be used to infer the presence of other clouds along the line of sight. Clearly, measurements of this sort are surely difficult to perform but, nevertheless, the possibility to have a free field of view exists. Finally, the whole absorption spectrum due to possibly intercepted galactic disk clouds should be less Doppler shifted (with respect to that due to halo clouds), because disk clouds are expected to have a slower velocity along the line of sight.

\subsection{Direct detection of MACHOs in M31 via infrared emission}

The possibility of direct detection of brown dwarfs via infrared searches has been carefully addressed by several authors [93, 94, 95, 96]. Evidently, the results of these studies automatically apply to MACHOs. In particular, we mention the recent analysis by Kerins and Carr [95] for a detailed discussion of the constraints that the infrared detector on ISO satellite (which has recently been launched) will set on our scenario. Actually, at variance with previous infrared observations done by IRAS satellite, ISO results will either lead to the direct discovery of MACHOs or put severe constraints on their properties.

Below, we shall consider the possibility of detecting MACHOs in M31 via their infrared emission [79].

For the sake of definiteness, we focus our attention on the case in which all MACHOs have equal mass $\sim 0.08 M_{\odot}$ and make up the fraction $(1-f)$ of the dark matter in M31. We suppose that all MACHOs have the same age $\sim 10^{10} \mathrm{yr}[93,94]$. As a consequence, MACHOs emit most of their radiation at the wavelength $\lambda_{\max } \sim 2.6 \mu \mathrm{m}$. Moreover, we assume that the M31 dark halo $(i)$ is made of uniformly distributed MACHOs, $(i i)$ is spherically symmetric and $(i i i)$ its mean density goes like $\rho(r)=\rho(0)\left[1+(r / a)^{2}\right]^{-1}$, where $r$ denotes here the distance of a MACHO from the M31 centre, $\rho(0)$ is the central dark matter density while $a$ is the dark matter core radius. As an approximation, we adopt for the latter two quantities the same values as for our galaxy. Typically, $a \sim 5 \mathrm{kpc}$ and $\rho(0) \sim 2.5 \times 10^{-24} \mathrm{~g} \mathrm{~cm}^{-3}$.

We are now in position to estimate the infrared surface brightness $I_{\nu}(b)$ of the M31 dark halo as a function of the projected separation $b$ (impact parameter) of a point in the halo from the M31 centre. Obviously, $I_{\nu}(b)$ is of direct relevance for pointed observations and we recall that it is overwhelmingly peaked around $\nu_{\max } \sim 11.5 \times 10^{13} \mathrm{~Hz}$. We can write [93]

$$
I_{\nu}(b)=\frac{(1-f)}{4 \pi} D \Gamma_{\nu} \rho(0) \mathcal{I}(b)
$$


where $\Gamma_{\nu}$ is the M31 dark halo specific luminosity. Furthermore, we have set

$$
\mathcal{I}(b)=\frac{1}{\rho(0) D} \int_{s_{\min }}^{s_{\max }} d s \rho(r(s))
$$

with $s$ denoting the distance of a generic M31 dark halo point from Earth along the line of sight. Manifestly, $s_{\min }$ and $s_{\max }$ are the nearest and the farthest intercepts of the M31 dark halo along the line of sight, respectively. It is very easy to rewrite eq.(37) as

$$
I_{\nu}(b) \sim 3.3 \times 10^{5}(1-f) \frac{x^{3}}{e^{x}-1} \mathcal{I}(b) \mathrm{Jy} \mathrm{sr}^{-1},
$$

with $x=\nu / 3.8 \times 10^{13} \mathrm{~Hz}$. As a matter of fact, the calculation of the dimensionless integral $\mathcal{I}(b)$ is straightforward and proceeds as in [97], leading to the result

$$
I_{\nu}(b) \sim 6.6 \times 10^{5} \frac{x^{3}}{e^{x}-1} \frac{a^{2}(1-f)}{D \sqrt{a^{2}+b^{2}}} \arctan \sqrt{\frac{L^{2}-b^{2}}{a^{2}+b^{2}}} \mathrm{Jy} \mathrm{sr}^{-1},
$$

where the M31 dark halo radius is taken to be $L \sim 50 \mathrm{kpc}$. Some numerical values of $I_{\nu_{\max }}(b)$ with $b=20$ and $40 \mathrm{kpc}$ are $\sim 2.1 \times 10^{3}(1-f) \mathrm{Jy} \mathrm{sr}^{-1}$ and $\sim 0.5 \times 10^{3}(1-f) \mathrm{Jy} \mathrm{sr}^{-1}$, respectively. For comparison, we recall that the halo of our galaxy would have in the direction of the galactic pole a luminosity $I_{\nu} \sim 2 \times 10^{3} \mathrm{Jy} \mathrm{sr}^{-1}$ at $3.8 \mu \mathrm{m}$ [93]. As is well known, the ISO Satellite contains an infrared array camera with expected sensitivity for extended sources of $\sim 1.7 \times 10^{4} \mathrm{Jy} \mathrm{sr}^{-1}$ per spatial resolution element in the wavelength range 3-6 $\mu \mathrm{m}$. Similarly, the planned SIRTF Satellite will contain an array camera with expected sensitivity of $\sim 1.7 \times 10^{3} \mathrm{Jy} \mathrm{sr}^{-1}$ per spatial resolution element in the wavelength range 2-6 $\mu \mathrm{m}$. Therefore - according to the above discussion - the MACHOs in the halo of M31 can be detected in the near future. In this connection, some points should be stressed. First, in order to disregard from eq.(40) contributions from ordinary stars in the M31 disc, the parameter $b$ should be $\geq 10 \mathrm{kpc}$. Second, the signal from the M31 halo can be identified and separated from the background via its b-modulation as dictated by eq.(40). Moreover, the infrared radiation originating from the MACHOs in the halo of our galaxy can be recognized (and subtracted) by its characteristic angular modulation.

So far, we have been concerned with the infrared detection of uniformly distributed MACHOs in M31. What about MACHOs clumped into dark clusters? The typical angular size of a dark cluster with radius $\sim 10 \mathrm{pc}$ in M31 is $\sim 3^{\prime \prime}$. This value is much too small to be resolved by the SIRTF detector, whose angular sensitivity is $\sim 7^{\prime \prime}$. This means that it will be impossible to distinguish an overall cluster-like MACHO from a uniform distribution. 
Finally, we point out that the angular size of dark clusters in the halo of our galaxy at a distance of $\sim 20 \mathrm{kpc}$ is $\sim 1.8^{\prime}$. In addition, the typical separation between two MACHO clusters is $\sim 14^{\prime}$ if their total number is $\sim 10^{6}$. As a result, a typical pattern of bright and dark spots should be seen by pointing the detector into different directions.

\section{Conclusions}

We have outlined a scenario in which dark clusters of MACHOs and cold molecular clouds naturally form in the galactic halo and we have addressed various observational tests for this model.

Halo molecular clouds would produce a $\gamma$-ray flux through the interaction with cosmic-ray protons, which at high galactic latitude is $\Phi_{\gamma}\left(90^{\circ}\right) \simeq \epsilon f 1.1 \times 10^{-6}$ photons $\mathrm{cm}^{-2} \mathrm{~s}^{-1} \mathrm{sr}^{-1}$. The detection of this background radiation can be performed by the GILDA detector on the planned Gamma Ray Astrophysics Mission, whose predicted sensitivity is a factor of 3 to 10 below the EGRET threshold [98, 99]. Molecular clouds in the M31 halo would yield an anisotropy $\Delta T / T \simeq 2 \times 10^{-5} f$ in the CBR and this effect might be measured by the detector on the planned satellite COBRAS/SAMBA ${ }^{14}$. A further method to discover halo molecular clouds is the presence of absorption lines in UV and optical spectra of the LMC stars near a previously microlensed one. Finally, MACHOs in the M31 halo can be detected via their emission in the infrared band. For pointed observations the maximum luminosity is $\sim 10^{3}(1-f) \mathrm{Jy} \mathrm{sr}^{-1}$ at $\lambda_{\max } \sim 2.6 \mu \mathrm{m}$.

Clearly, it turns out that the available observations are not yet sensitive enough to either confirm or rule out our picture, especially as far as molecular clouds are concerned. Yet, from the above discussion we see with satisfaction that our proposal may well be tested in the near future. Whatever the outcome will be, it seems important to us that this issue should be settled.

We would like to close this paper by suggesting that the considered scenario might also hold for elliptical galaxies, for which there is positive evidence in favour of dark matter (see e.g. [101]). Also in this case, the dark matter can be in the form of dark clusters of MACHOs and molecular clouds, which are not destroyed by the X-ray flux present in ellipticals (whose

\footnotetext{
${ }^{14}$ Private communication from M. Signore [100].
} 
intensity is always less than $\sim 10^{43} \mathrm{erg} \mathrm{s}^{-1}$ ). An advantage of this idea is that the diffuse gas observed in clusters of galaxies can be understood as arising from the tidal stripping from neighboring galaxies. This fact naturally explains the ROSAT observation that $\sim 30 \%$ of the dynamical mass in clusters of galaxies is in baryonic form [102]. Remarkably enough, these speculations would lead to a unified picture of galaxy formation (the parameter which mainly discriminates between either spirals or ellipticals would be the initial total angular momentum).

\section{Acknowledgements}

We thank G. Barbiellini, B. Bertotti, B. Carr, F. D'Antona, K. Griest, J. Lequeux, D. Pfenniger and A. Qadir for useful suggestions. FDP and GI have been partially supported by Italian Space Agency (ASI) and PJ by the Swiss National Science Foundation.

\section{References}

[1] S.M. Faber and J.S. Gallagher, Ann. Rev. Astron. Astrophys. 17, 135 (1979).

[2] V. Trimble, Ann. Rev. Astron. Astrophys. 25, 425 (1987).

[3] V.C. Rubin, W.K. Ford and N. Thonnard, Astrophys. J. 238, 471 (1980).

[4] R. Sancisi and T.S. van Albada, in: Observational Cosmology, IAU Symposium 124 (Reidel Publishing Company, Dordrecht, 1987) and in: Dark Matter in the Universe, IAU Symposium 117, ed. by Kormandy and Knapp (Reidel Publishing Company, Dordrecht, 1987).

[5] B.J. Carr, Ann. Rev. Astron. Astrophys. 32, 531 (1994).

[6] C. J. Copi, D. N. Schramm and M. S. Turner, Science 267, 192 (1995).

[7] A. De Rújula, Ph. Jetzer and E. Massó, Astron. and Astrophys. 254, 99 (1992).

[8] B. Paczyński, Astrophys. J. 304, 1 (1986).

[9] A. De Rújula, Ph. Jetzer and E. Massó, Mont. Not. R. Astr. Soc. 250, 348 (1991)

[10] K. Griest, Astrophys. J. 366, 412 (1991). 
[11] R.J. Nemiroff, Astron. and Astrophys. 24773 (1991).

[12] E. Aubourg et al., Nature (London) 365, 623 (1993).

[13] R. Ansari et al., submitted to Astron. and Astrophys. (1995).

[14] C. Alcock et al., Nature (London) 365, 621 (1993) and Astrophys. J. 445, 133 (1995);

D. Bennett et al., astro-ph 9510104.

[15] A. Udalski et al., Acta Astronomica 43, 289 (1993) and Acta Astronomica 44, 165 and 227 (1994).

[16] C. Alcock et al., astro-ph 9512146.

[17] C. Alard et al., paper in preparation.

[18] B. Paczyński, Astrophys. J. 371, L63 (1991).

[19] K. Griest et al., Astrophys. J. 372, L79 (1991).

[20] H. Zhao, D. Spergel and M. Rich, Astrophys. J. 440, L13 (1995).

[21] B. Paczyński et al., Astrophys. J. 435, L113 (1995).

[22] K. C. Sahu, Nature (London) 370, 275 (1994).

[23] X. P. Wu, Astrophys. J. 435, 66 (1994).

[24] A. Gould, Astrophys. J. 441, 77 (1995).

[25] A. Gould, J. Miralda-Escudé and J.N. Bahcall, Astrophys. J. 423, L105 (1994).

[26] E.M. Hu, J.S. Huang, G. Gilmore and L.L. Cowie, Nature 371, 493 (1994).

[27] G.F. Giudice, S. Mollerach and E. Roulet, Phys. Rev. D50, 2406 (1994).

[28] C. Alcock et al., Phys. Rev. Lett. 74, 2867 (1995) and Astrophys. J., in press.

[29] F. De Paolis, G. Ingrosso and Ph. Jetzer, astro-ph 9510099.

[30] Ph. Jetzer and E. Massó, Phys. Lett. B323, 347 (1994); Ph. Jetzer, Astrophys. J. 432, L43 (1994). 
[31] E. Aubourg et al., Astron. and Astrophys. 301, 1 (1995).

[32] F. De Paolis, G. Ingrosso, Ph. Jetzer and M. Roncadelli, Phys. Rev. Lett. 74, 14 (1995).

[33] F. De Paolis, G. Ingrosso, Ph. Jetzer and M. Roncadelli, Astron. and Astrophys. 295, 567 (1995).

[34] F. De Paolis, G. Ingrosso, Ph. Jetzer and M. Roncadelli, Comments on Astrophys. 18, 87 (1995).

[35] K.M. Ashman and B.J. Carr, Mon. Not. R. Astr. Soc. 234, 219 (1988) and 249, 13 (1991).

[36] B. J. Carr and C. G. Lacey, Astrophys. J. 316, 23 (1987).

[37] K. M. Ashman, Mon. Not. R. Astr. Soc. 247, 662 (1990).

[38] D. Eichler and J. Silk, Science 257, 937 (1992); B. Moore and J. Silk, Astrophys. J. 442, L5 (1995).

[39] I. Wasserman and E.E. Salpeter, Astrophys. J. 433, 670 (1994).

[40] O.E. Gerhard and J. Silk, astro-ph 9509149.

[41] D. Pfenniger, F. Combes and L. Martinet, Astron. and Astrophys. 285, 79 (1994); D.

Pfenniger and F. Combes, Astron. and Astrophys. 285, 94 (1994).

[42] N.A. Bahcall, L.M. Lubin and V. Dorman, Astrophys. J. 447, L81 (1995).

[43] A.C. Fabian, Ann. Rev. Astron. Astrophys. 32, 277 (1994).

[44] J. Lequeux, R. J. Allen and S. Guilloteau, Astron. and Astrophys. 280, L23 (1993).

[45] D.T. Frayer, R.L. Brown and P.A. Vanden Bout, Astrophys. J. 433, L5 (1994).

[46] B.J. Weiner and T.B. Williams, astro-ph 9512017.

[47] S. M. Fall and M. J. Rees, Astrophys. J. 298, 18 (1985).

[48] H. Kang, P. R. Shapiro, S. M. Fall and M. J. Rees, Astrophys. J. 363, 488 (1990). 
[49] S. D. Murray and D. N. C. Lin, Astrophys. J. 339, 933 (1989).

[50] F. Palla, E. E. Salpeter and S. W. Stahler, Astrophys. J. 271, 632 (1983).

[51] G. Einaudi and A. Ferrara, Astrophys. J. 371, 571 (1991).

[52] A. Dalgarno and R. A. McCray, Ann. Rev. Astron. Astrophys. 10, 375 (1972).

[53] B.J. Carr, J. Bond and W. Arnett, Astrophys. J. 277, 445 (1984).

[54] M. Vietri and E. Pesce, Astrophys. J. 442, 618 (1995).

[55] F. Palla and E. E. Stahler, in Galactic and Extragalactic Star Formation, ed. by R.E. Pudeitz and M. Fich, NATO ASI Ser. C 232 (Kluwer Academic Publishers, Dordrecht, 1988) p. 519.

[56] F. D'Antona and I. Mazzitelli, Ann. Rev. Astron. Astrophys. 28, 139 (1990).

[57] R. Rebolo, M.R. Zapatero Osorio and E.L. Martin, Nature 377, 129 (1995); T. Nakajima et al., Nature 378, 463 (1995).

[58] J. M. Scalo, in Protostars and planets II, ed. by D.C. Black and M.S. Mathews (University of Arizona Press, Tucson, 1985), p. 201.

[59] J.M. Dickey and F.J. Lockman, Ann. Rev. Astron. Astrophys. 28, 215, (1990).

[60] E.F. van Dishoeck and J.H. Black, Astrophys. J. Suppl. 62, 109 (1986).

[61] B.G. Elmegreen, Astrophys. J. 338, 178 (1989).

[62] J. Binney and S. Tremaine, Galactic Dynamics (Princeton University Press, Princeton, 1987).

[63] E.F. van Dishoeck, in Astrochemistry, IAU Symp. 120, ed. by M.S. Vardya and S.P. Tarafdar (Reidel Publishing Company, Dordrecht, 1987), p. 51.

[64] Y.P. Viala, H. Abgrall and E. Roueff, in Astrochemistry, IAU Symp. 120, ed. by M.S. Vardya and S.P. Tarafdar (Reidel Publishing Company, Dordrecht, 1987), p. 227. 
[65] T.J. Millar, J.M.C. Rawlings, A. Bennett, P.D. Brown and S.B. Charnley, Astron. and Astrophys. Suppl. 87, 585 (1991).

[66] C.P. O'Dea et al., Astrophys. J. 422, 467 (1994).

[67] R. Morrison and D. McCammon, Astrophys. J. 270, 119 (1983).

[68] P.F. Goldsmith and W.D. Langer, Astrophys. J. 222, 881 (1978).

[69] E. Maoz, Astrophys. J. 428, L5 (1994).

[70] D. Breitschwerdt, J.F. McKenzie and H.J. Völk, Astron. and Astrophys. 245, 79 (1991).

[71] K.R. Lang, Astrophysical Formulae (Springer-Verlag, Berlin, 1974).

[72] T.K. Gaisser, in Cosmic Rays and Particle Physics (Cambridge University Press, Cambridge, 1990).

[73] C. Forti et al., Phys. Rev D42, 3668 (1990).

[74] V.S. Berezinskii et al., Astrophysics of cosmic rays (North-Holland, Amsterdam, 1990).

[75] Particle Data Group, Phys. Lett. B239 (1990).

[76] L. Danly, C. E. Albert and K. D. Kuntz, Astrophys. J. 416, L29 (1993).

[77] A. Bouquet, P. Salati and J. Silk, Phys. Rev. D40, 3168 (1989).

[78] P. Salati, P. Chardonnet, X. Luo, J. Silk and R. Taillet, preprint ENSLAPP-A-528 (1995).

[79] F. De Paolis, G. Ingrosso, Ph. Jetzer, A. Qadir and M. Roncadelli, Astron. and Astrophys. 299, 647 (1995).

[80] L. Toffolatti et al., Astrophys. Lett. and Comm, in press (1994).

[81] R. Maoli, F. Melchiorri and D. Tosti, Astrophys. J. 425, 372 (1994).

[82] R.A. Sunyaev and Ya.B. Zeldovich, Annual. Rev. Astron. Astrophys. 18, 537 (1980).

[83] B. Melchiorri and F. Melchiorri, Rivista del Nuovo Cimento 17, 1 (1994). 
[84] E.J.A. Meurs and R.T. Harmon, Astron. and Astrophys. 206, 53 (1988).

[85] R.A.M. Walterbos and P.B.W. Schwering, Astron. and Astrophys. 180, 27 (1987).

[86] F. De Paolis, G. Ingrosso, Ph. Jetzer and M. Roncadelli, Astrophys. and Space Science, in press.

[87] L. Pasquini, J. Storm and H. Dekker, Eso Messenger 77, 5 (1994).

[88] J.C. Brandt, in The Space Telescope Observatory, NASA CP-2244, ed. by D.N.B. Hall, (1982) p. 76.

[89] L. Spitzer, in Physical Processes in the Interstellar Medium (John Wiley, New York, 1978).

[90] B.T Draine and H.M. Lee, Astrophys. J. 285, 89 (1984).

[91] C. Blaha and R.M. Humphreys, Astr. J. 98, 1598 (1989).

[92] M.A. Dopita in Star Forming Regions, IAU Symp. 115, ed. by M. Peimbert and J. Jugaku (Reidel Publishing Company, Dordrecht, 1987), p. 501.

[93] F.C. Adams and T.P. Walker, Astrophys. J. 359, 57 (1990).

[94] R.A. Daly and G.C. McLaughlin, Astrophys. J. 390, 423 (1992).

[95] E.J. Kerins and B.J. Carr, Mont. Not. R. Astr. Soc. 266, 775 (1994).

[96] L.A. Nelson, S. Rappaport and P.C. Joss, Astrophys. J. 404, 723 (1993).

[97] Ph. Jetzer, Astron. and Astrophys. 286, 426 (1994).

[98] A. P. Marscher et al. preprint, submitted in response to NRA 94-OSS-15 (1994).

[99] G. Barbiellini et al., Nucl. Instr. and Meth. A354, 547 (1995).

[100] M. Signore et al. preprint, SISSA 111/95/A.

[101] F. De Paolis, G. Ingrosso and F. Strafella, Astrophys. J. 438, 83 (1995).

[102] U.G. Briel, J.P. Henry and H. Böhringer, Astron. and Astrophys. 259, L31 (1992). 
[103] K. Kirby and A. Dalgarno, Astrophys. J. 224, 444 (1978).

[104] D.C. Morton and L. Noreau, Astrophys. J. Suppl. 95, 301 (1994).

[105] C.P. de Vries and E.F. van Dishoeck, Astron. and Astrophys. 203, L23 (1988).

[106] R. Gredel, E.F. van Dishoeck and J.H. Black, Astron. and Astrophys. 251, 625 (1991). 
Table 1: Some possible molecules in halo clouds: lower limit of the molecular fractional abundance (with respect to $\mathrm{H}_{2}$ ) to have optically thick lines, oscillator strengths (whose values are taken from $[103,104,105,106]$ ), classification and wavelengths of some optically thick vlines in optical and UV bands.

\begin{tabular}{ccccrc}
\hline Molecule & Abundance & $f_{j k}$ & Transition & Band & $\lambda(\AA)$ \\
\hline$L i^{7} H^{1}$ & $10^{-11}$ & $9.88 \times 10^{-3}$ & $A^{1} \Sigma^{+}-X^{1} \Sigma^{+}$ & $(6,0)$ & 3638.02 \\
& & $1.07 \times 10^{-2}$ & & $(7,0)$ & 3634.89 \\
& & & & $(8,0)$ & 3649.10 \\
\hline$C^{12} O^{16}$ & $1.04 \times 10^{-2}$ & & & \\
& & $2.91 \times 10^{-2}$ & $A^{1} \Pi-X^{1} \Sigma^{+}$ & $(1,0)$ & 1509.75 \\
& & $4.01 \times 10^{-2}$ & & $(2,0)$ & 1477.57 \\
& & $3.46 \times 10^{-2}$ & & $(3,0)$ & 1447.35 \\
\hline$C^{12} H^{1}$ & $10^{-10}$ & $5.06 \times 10^{-3}$ & $A^{2} \Delta-X^{2} \Pi$ & $(0,0)$ & 4300.30 \\
& & $1.61 \times 10^{-3}$ & & $(1,0)$ & 3846.25 \\
\hline$C^{12} N^{14}$ & $10^{-12}$ & $3.38 \times 10^{-2}$ & $B^{2} \Sigma^{+}-X^{2} \Sigma^{+}$ & $(0,0)$ & 3874.61 \\
\hline
\end{tabular}

\section{Figure Captions}

Fig. 1: Schematic representation of the proposed experiment [79]. A pair of anisotropy measuring horns of $1^{0}$ resolution - separated by an angle of $4^{0}$ - are centered on M31, whose core has an angular diameter of $\sim 1^{0}$ as seen from Earth. The horns would be rotated about their axis in order to scan the annular ring in 180 steps, each of $1^{0}$. 


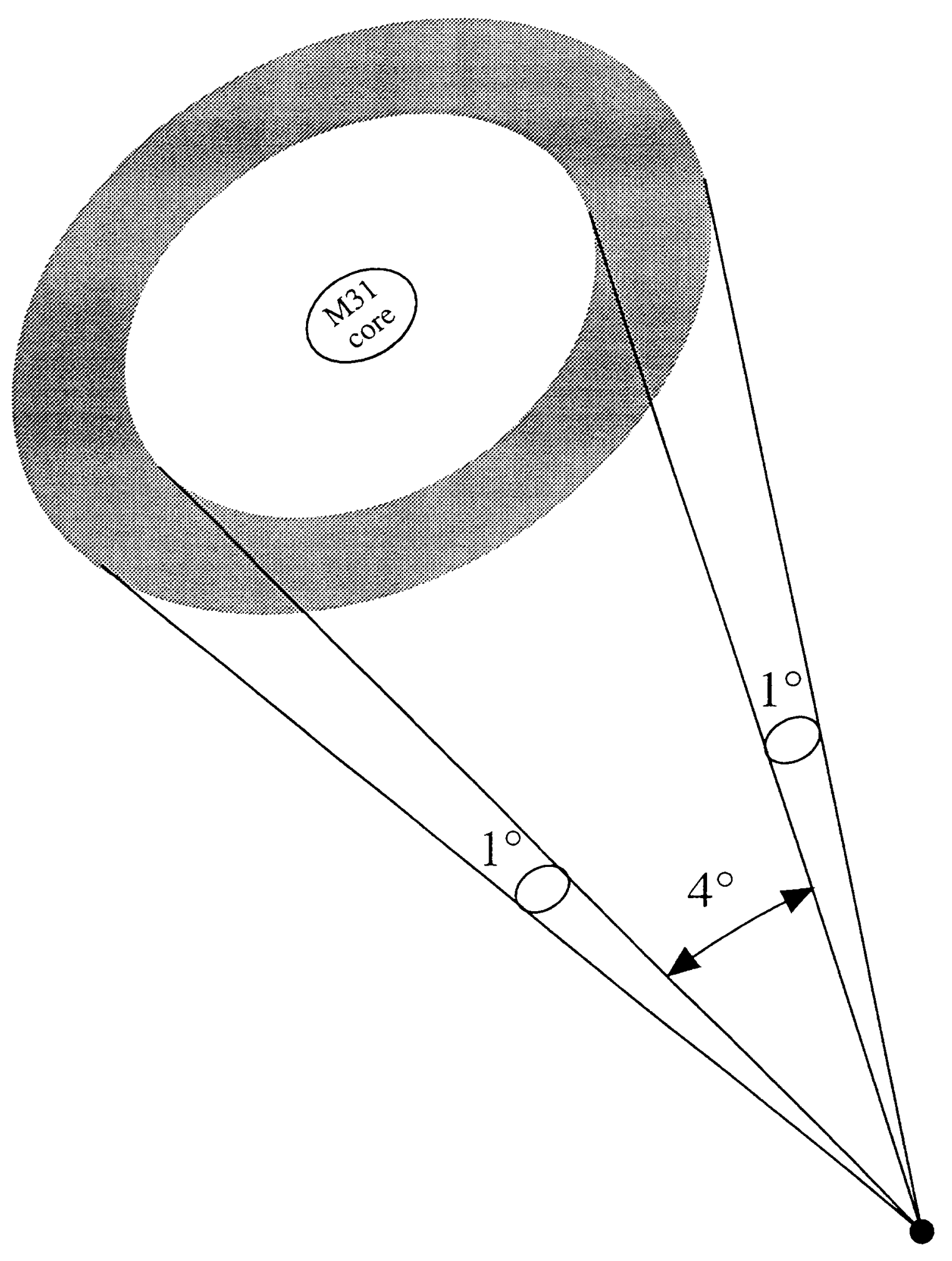


